\title{
Isolation Of Potential Pathogenic Bacteria From Nasopharynx From Patients Having Cough For More Than Two Weeks.
}

\author{
Kazi Yesmin ${ }^{1}$, K Z Mamun², SM Shamsuzzaman², Akhtaruzzaman Chowdhury², Khadeza Khatun², Jahangir Alam². \\ ${ }^{1}$ Assistant Professor, Dept of Microbiology, Green Life Medical College, Dhaka; ${ }^{2}$ Dept. of Microbiology, Dhaka Medical College, \\ Dhaka; ${ }^{3}$ Medical Officer, UHC Kishorgonj, Nilphamari;
}

\begin{abstract}
s
This cross sectional study was done from January 2009 to June 2010 in Microbiology department of Dhaka Medical College on isolation of potential pathogenic bacteria from nasopharynx from patients having cough for more than two weeks. The nasopharyngeal swabs were collected from 290 patients for culture and 101 blood samples were collected for slide agglutination test against anti- Bordetella antibody. In case of culture of nasopharyngeal swabs, 117 (73.12\%) samples yielded growth of single organism, $16(10 \%)$ yielded mixed growth and $27(16.88 \%)$ samples yielded no growth in children. In adults, $85(65.39 \%)$ samples yielded growth of single organism, $1(0.77 \%)$ yielded mixed growth and 44 (33.84\%) samples yielded no growth. Highest incidence (33.33\%) of S. pneumoniae was found in age group of 1-5 years, highest incidence $(32.20 \%)$ of $\mathrm{N}$. meningitidis was found in age group of 18-25 years and highest incidence (32\%) of Staph. aureus was found in age group of $25-50$ years. One $(0.63 \%)$ B. pertussis was isolated from a child and $5(3.84 \%)$ B. parapertussis were isolated from adults. In this study, $116(83.45 \%)$ samples yielded growth in lower socioeconomic group and $101(\mathbf{7 0 . 6 2} \%)$ yielded growth in the samples of middle socioeconomic group. Among the study participants, 31(30.70\%) were positive and $70(69.30 \%)$ were negative for antibody against B. pertussis. In anti-Bordetella antibody positive cases, $14(93.33 \%)$ children had duration of cough for $2-3$ weeks, 7 (43.75\%) adults had duration of cough for 3-4 weeks and 1 (6.25\%) adults had more than 6 weeks.
\end{abstract}

Key words: Potential pathogenic bacteria, nasopharyngeal swabs, Bordetella species, Charcoal blood agar media.

\section{Introduction}

Infections of the respiratory tract is a major cause of morbidity and mortality throughout the world ${ }^{1}$. Cough is the most frequent symptom of respiratory infection of which about $12 \%$ of the general population have chronic cough. Pertussis infection is one of the causes of persistent cough. Approximately $13 \%-20 \%$ have cough in adolescents and adults due to $\mathrm{B}$. pertussis infection ${ }^{2-3}$. Adults with waning

\section{Correspondence:}

Dr. Kazi Yesmin

Assistant Professor

Department of Microbiology, Green Life Medical College

Dhaka

Phone : 01716-063969 vaccine-induced immunity are increasing and also suffering from pertussis ${ }^{4}$. Recently, an increase in reported cases of pertussis in adolescents and adults has been noted in many countries despite high immunization rates in children. The clinical presentation in adolescents, adults and vaccinated individuals may be atypical, with paroxysmal cough of short duration or simply a persistent cough ${ }^{5-6}$. The affected adolescents and adults act as reservoirs of the disease to the vulnerable population of infants ${ }^{7}$. The nasopharynx is densely colonized by a broad variety of microorganisms, including commensal bacteria as well as potential pathogens. However, when the condition of the host is altered, microorganism may invade adjacent sites and/or invade the bloodstream, causing disease. On the other hand, the 
nasopharynx is a major source of secretions containing bacteria that spread between individuals and may become pathogens ${ }^{8}$.

\section{Material and Methods:}

This cross sectional study included 290 patients of all age groups attending at Medicine and Pediatrics OPD of Dhaka Medical College and Dhaka Shishu Hospital with symptom of cough for more than two weeks. Data regarding age, sex, monthly income, level of education, DPT vaccination and duration of cough were collected by using a predesigned data collection sheet. Specimens of nasopharyngeal swab were collected and were plated immediately in different culture media. All the Charcoal blood agar media were incubated at $35^{\circ} \mathrm{C}$ in aerobic condition up to 7 days and adequate humidity was maintained by placing a flask of water on the floor of incubator and inspection was done regularly every morning. All organisms were identified by their colony morphology, staining characteristic, hemolysis on blood agar, pigment production, motility and other relevant biochemical tests as per standard methods. Isolated organism of B . pertussis was confirmed by using specific anti- B. pertussis antiserum. Detection of Anti-B. pertussis IgGIIgM antibody in serum by slide agglutination test.

\section{Result}

A total of 290 patients with history of cough for more than 2 weeks were selected for the study. The highest number of patients were $48(16.55 \%)$ in the age group of 1-4 years followed by $45(15.52 \%)$ in the age group of 5-9 years (Table I).

Figure 1. shows the sex distribution of the study population. Among 290 patients, 122 (42\%) were males and 168 (58\%) were females with a male female ratio of $3: 4$.

Table III shows the distribution of the study subjects by duration of cough. Among the participants 158 (54.50\%) had cough for 2-3 weeks, 75 (25.90\%) had cough for 3-4 weeks, $21(7.20 \%)$ had cough for 5 - 6 weeks and $36(12.40 \%)$ had cough for more than 6 weeks.

Table IV shows the distribution of the respondents by history of vaccination against pertussis. Among the study subjects $183(63.10 \%)$ received vaccination, $60(20.70 \%)$ didn't receive the vaccination and $47(16.20 \%)$ couldn't recall if they had vaccination.

Table V shows the vaccination status against pertussis (DPT) among the 183 participants with history of vaccination, 148
(80.90\%) had completed the full course of vaccination, 20 $(10.90 \%)$ completed up to $2^{\text {nd }}$ dose, $15(8.02 \%)$ received only the $1^{\text {st }}$ dose and $2(1.09 \%)$ cases did not complete vaccination schedule.

Table VIII shows results of culture of nasopharyngeal swab. In children, 117 (73.12\%) samples yielded growth of single organism, $16(10 \%)$ yielded mixed growth and $27(16.88 \%)$ samples yielded no growth. In adults, $85(65.39 \%)$ samples yielded growth of single organism, $1(0.77 \%)$ yielded mixed growth and 44 (33.84\%) samples yielded no growth. P statistically significant.

Table IX shows isolation of different organisms among children and adults. The most common isolated organism was Neisseria among children $(\mathrm{n}=69 ; 43.12 \%)$ and Staphylococcus among adults $(n=43 ; 33.08 \%)$.

Table $\mathrm{X}$ shows distribution of growth according to age groups. Among the study population, highest incidence (33.34\%) of S. pneumoniae was found in age group of 1-5 years and highest incidence (32.20\%) of N. meningitidis was found in age group of 18-25 years and highest incidence (32\%) of Staph. aureus was found in age group of 25-50 years.

Table XI shows distribution of socioeconomic condition and isolation of organisms. Among the nasopharyngeal samples of study population, $116(83.45 \%)$ samples yielded growth and $23(16.55 \%)$ samples yielded no growth in lower socioeconomic condition. One hundred one $(70.63 \%)$ yielded growth and $42(29.37 \%)$ yielded no growth in the samples of study population of middle socioeconomic. P statistically significant.

Table XII shows the distribution of study participants by serology result. Among the study participants, 31(30.70\%) were positive and $70(69.30 \%)$ were negative for antibody against B. pertussis.

Table XIII shows duration of cough among anti-Bordetella antibody positive cases. Among study population, 14 (93.33\%) children had duration of cough for 2-3 weeks, 7 $(43.75 \%)$ adults had duration of cough for 3-4 weeks and 1 $(6.25 \%)$ adults had more than 6 weeks. 
Table I: Distribution of the study participants by age ( $\mathrm{n}$ $=290$ )

\begin{tabular}{lll}
\hline Age group & Frequency & Percentage \\
\hline$<1$ years & 37 & 12.75 \\
$1-4$ years & 48 & 16.55 \\
$5-9$ years & 45 & 15.52 \\
$10-18$ years & 30 & 10.34 \\
$19-29$ years & 40 & 13.79 \\
$30-39$ years & 20 & 06.81 \\
$40-49$ years & 27 & 09.33 \\
$50-59$ years & 17 & 05.84 \\
$>=60$ years & 26 & 09.06 \\
Total & 290 & 100.0
\end{tabular}

Figure 1: Distribution of the study participant's by sex

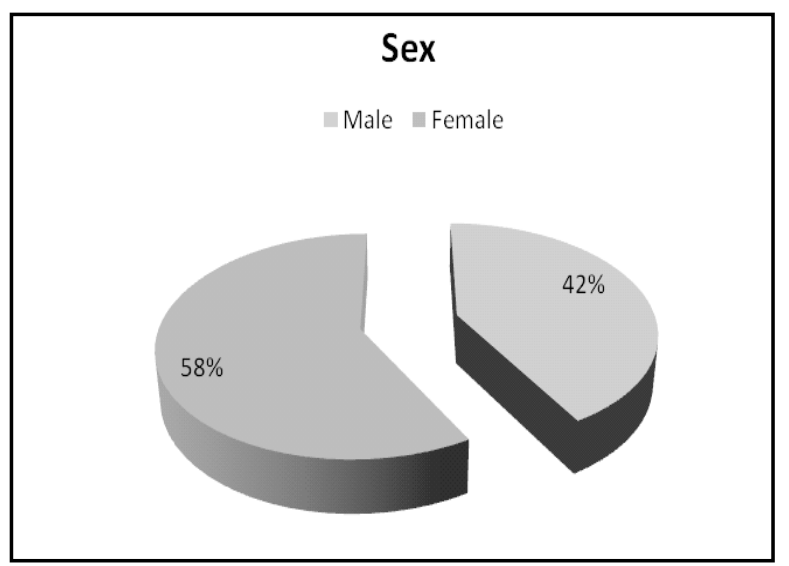

Table III: Duration of cough among the study population

\begin{tabular}{lll}
\hline Duration of cough & Frequency & Percentage \\
\hline 2 - 3 weeks & 158 & 54.50 \\
3 - 4 weeks & 75 & 25.90 \\
5 - 6 weeks & 21 & 7.20 \\
$>$ 6 weeks & 36 & 12.40 \\
Total & 290 & 100.00 \\
\hline
\end{tabular}

Table IV: History of DPT vaccination

\begin{tabular}{lll}
\hline $\begin{array}{l}\text { History of } \\
\text { vaccination }\end{array}$ & Frequency & Percentage \\
\hline Vaccinated & 183 & 63.10 \\
Non- vaccinated & 60 & 20.70 \\
Couldn't recall & 47 & 16.20 \\
Total & 290 & 100.00 \\
\hline
\end{tabular}

Table V: Status of vaccination among 183 population

\begin{tabular}{lll}
\hline Status of vaccination & Frequency & Percentage \\
\hline 1 dose & 15 & 08.20 \\
2 doses & $18+2^{*}$ & 10.90 \\
3 doses & 148 & 80.90 \\
Total & 183 & 100.00 \\
\hline
\end{tabular}

*2 cases did not complete vaccination schedule.

Table VIII: Results of culture of nasopharyngeal swab $(\mathrm{n}=290)$

\begin{tabular}{lcccc}
\hline & No growth & Single growth & Mixed growth & Total \\
\hline Children & $27(16.88)$ & $117(73.12)$ & $16(10.00)$ & $160(100)$ \\
Adults & $44(33.84)$ & $85(65.39)$ & $1(0.77)$ & $130(100)$ \\
Total & $71(24.48)$ & $202(69.66)$ & $17(5.86)$ & $290(100)$ \\
\hline
\end{tabular}

Table IX: Isolation of different organisms among children and adults

\begin{tabular}{lllllll}
\hline & \multicolumn{3}{c}{ Children } & \multicolumn{3}{c}{ Adults } \\
Growth & Single Mixed & Total & Single & Mixed & Total \\
\hline B. pertussis & 1 & 0 & $1(0.63)$ & 0 & 0 & $0(0.00)$ \\
B. parapertussis & 0 & 0 & $0(0.00)$ & 5 & 0 & $5(3.84)$ \\
Staph. aureus & 5 & 4 & $9(5.62)$ & 16 & 0 & $16(12.31)$ \\
S. pneumoniae & 15 & 2 & $17(10.62)$ & 4 & 0 & $4(3.08)$ \\
N. meningitidis & 25 & 3 & $28(17.50)$ & 3 & 0 & $3(2.31)$ \\
H. influenzae & 1 & 0 & $1(0.63 \%)$ & 0 & 0 & $0(0.0)$ \\
Staphylococcus (Coag -ve) & 12 & 10 & $22(13.75)$ & 26 & 1 & $27(20.77)$ \\
Streptococcus (others) & 24 & 6 & $30(18.75)$ & 14 & 0 & $14(10.77)$ \\
Neisseria (others) & 34 & 7 & $41(25.62)$ & 16 & 1 & $17(13.08)$ \\
\hline
\end{tabular}

Figures in parentheses represent percentage Note- Streptococcus (other) - Other than S. pneumoniae Neisseria (others) - Other than N. meningitides 
Table X: Distribution of growth according to age groups

\begin{tabular}{lllllllll}
\hline Growth & $<$ lyear & $1-5$ years & $5-10$ years & $10-18$ years & $18-25$ years & $25-50$ years $>50$ years & Total \\
\hline S. pneumoniae & $6(28.58)$ & $7(33.34)$ & $2(9.52)$ & $2(9.52)$ & $2(9.52)$ & $0(0.0)$ & $2(9.52)$ & $21(100)$ \\
N. meningitidis & $1(3.22)$ & $3(9.78)$ & $5(16.12)$ & $8(25.80)$ & $10(32.20)$ & $2(6.44)$ & $2(6.44)$ & $31(100)$ \\
Staph. aureus & $1(4)$ & $2(8)$ & $2(8)$ & $3(12)$ & $5(20)$ & $8(32)$ & $4(16)$ & $25(100)$ \\
Neisseria- others $7(12.06)$ & $15(25.8)$ & $6(10.35)$ & $8(13.80)$ & $10(17.24)$ & $8(13.80)$ & $4(6.89)$ & $58(100)$ \\
Streptococcus-others $8(18.18)$ & $8(18.18)$ & $5(11.37)$ & $8(18.18)$ & $3(6.82)$ & $5(11.37)$ & $7(15.90)$ & $44(100)$ \\
Staphylococcus & $5(10.20)$ & $8(16.35)$ & $4(8.16)$ & $5(10.20)$ & $5(10.20)$ & $17(34.69)$ & $5(10.20)$ & $49(100)$ \\
Coa(-)ve & & & & & & &
\end{tabular}

Figures in parentheses represent percentage

Table XI: Isolation of organisms in different socioeconomic groups $(\mathrm{n}=290)$

\begin{tabular}{llll}
\hline $\begin{array}{l}\text { Socioeconomic } \\
\text { condition }\end{array}$ & \multicolumn{3}{c}{ Isolation } \\
& No growth & Growth & Tota \\
\hline Low income group & $23(16.55)$ & $116(83.45)$ & $139(100)$ \\
Middle income group & $42(29.37)$ & $101(70.63)$ & $143(100)$ \\
High income group & $6(75)$ & $2(25)$ & $8(100)$ \\
Total & $71(24.49)$ & $219(75.51)$ & $290(100)$ \\
\hline
\end{tabular}

Figures in parentheses represent percentage

Table XII: Results of anti-Bordetella antibody test in children and adults $(\mathrm{n}=101)$

\begin{tabular}{llll}
\hline & Children & Adults & Total \\
\hline Serology positive & $15(30)$ & $16(31.38)$ & $31(30.70)$ \\
Serology negative & $35(70)$ & $35(68.62)$ & $70(69.30)$ \\
Total & $50(100)$ & $51(100)$ & $101(100)$ \\
\hline
\end{tabular}

Figures in parentheses represent percentage

Table XIII: Duration of cough among anti-Bordetella antibody positive cases $(\mathrm{n}=101)$

\begin{tabular}{llllll}
\hline & 2-3 weeks & $3-4$ weeks & $5-6$ weeks & $>6$ weeks & Total \\
\hline Children & $14(93.33)$ & $1(6.67)$ & $0(0.00)$ & $0(0.00)$ & $15(100)$ \\
Adults & $5(31.25)$ & $7(43.75)$ & $3(18.75)$ & $1(6.25)$ & $16(100)$
\end{tabular}

Figures in parentheses represent percentage

Chi-square -9.04 , df 3, p $=0.023$ (statistically significant)

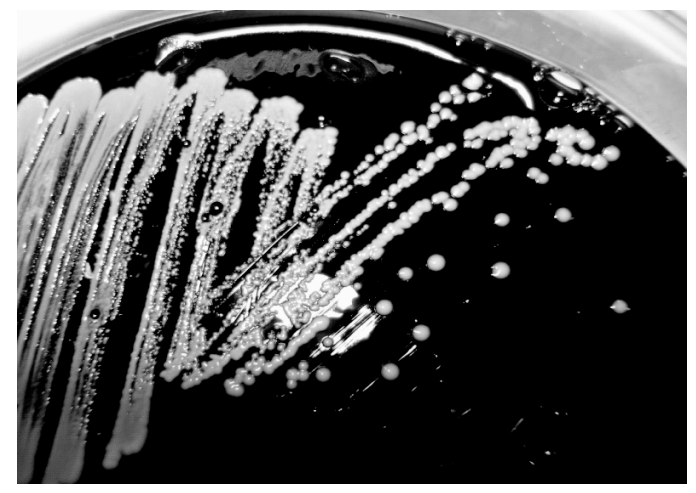

Fig-5: Pure culture of Bordetella spp. in the charcoal blood agar media.

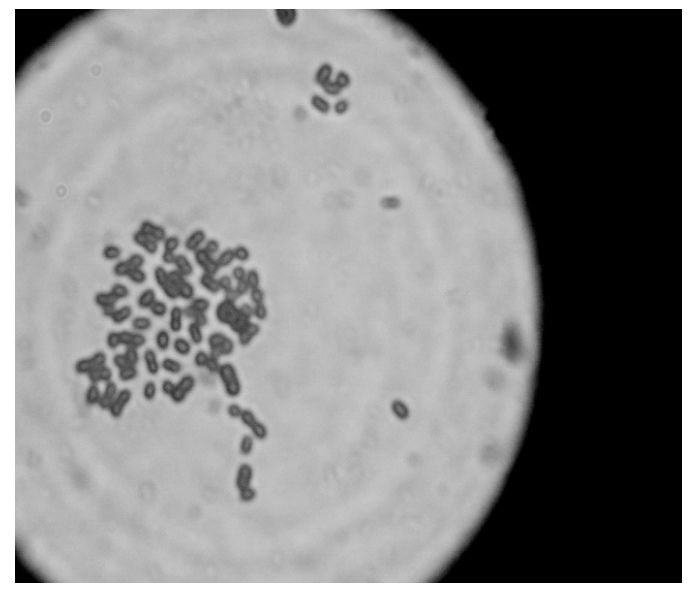

Fig 6: Gram stained smear showing Bordetella spp. from culture.

\section{Discussion}

In the present study, culture of 290 nasopharyngeal swabs samples yielded growth in 219 (75.51\%) samples. In children, $73.12 \%$ yielded growth, mixed growth was $10 \%$ and no growth was $16.88 \%$ (Table-VIII). In adults, $65.39 \%$ yielded growth, mixed growth was $0.77 \%$ and no growth was $33.84 \%$ (Table-VIII). Isolation of organisms was more in children than adults. This difference was statistically significant $(\mathrm{p}=$ 0.047). Carriage of poentially pathogenic bacteria decreased with age in this study. In Spain, Garcia-Rodriguez and Martinez (2002) reported that the prevalence of carriage of pathogenic bacteria in healthy individuals decreased with age ${ }^{9}$. These bacteria are often present in nasopharyngeal samples taken from healthy children, but rarely from people of more than 16 years of age.

In this study, Staph. aureus was isolated in $12.31 \%$ adults and $5.62 \%$ children. In Sweden, Nilsson and Ripa (2006) reported that the rate of Staph. aureus isolation from anterior nares were $31 \%$ from patients admitted to an orthopedic ward ${ }^{10}$. In this study, Staph. aureus was isolated in 20\% among 18-25 
years and $32 \%$ among $25-50$ years. Teenagers and male individuals were more likely to be carriers of Staph. aureus ${ }^{11}$. Melles et al. (2006) demonstrated that passive smoking showed a significant association with carriage of a specific Staph. Aureus ${ }^{12}$.

Among the study population, S. pneumoniae was isolated in $10.62 \%$ children and $3.08 \%$ adults (Table-IX). In Sweden, Gunnarsson et al. (1996) reported that isolation of S. pneumoniae was $6-19 \%$ in healthy children and $0.8 \%$ in healthy adults ${ }^{13}$. Syrjanen et al. (2001) had shown that carriage of S. pneumoniae increased during respiratory tract infections ${ }^{14}$. In the present study, peak incidence $(33.33 \%)$ of S. pneumoniae was found in the age group of one to 5 years, followed by $28.57 \%$ among children less than one year (Table-X). In Mexico, Butler et al. (2007) reported that 29.9\% S. pneumoniae was isolated from subjects aged 2 months to 6 years ${ }^{15}$.

In the present study, $\mathrm{N}$. meningitidis was isolated in $17.50 \%$ children and $2.31 \%$ adults (Table-X). Similar high prevalence was observed by Cunningham et al. (2001) and reported that pharyngeal carriage of N. meningitides was $10-23 \%$ from direct plating of nasopharyngeal swabs ${ }^{16}$. In this study, carriage rate was $3.22 \%$ in less than one year age group and increased up to $42 \%$ in the age group of 5-18 years and $18-25$ years and then decreased to $6.44 \%$ in older age groups. Blackwell et al. (1990) reported that the carriage rate was shown to be less than 3\% in children younger than 4 years and increased to $24-37 \%$ in the age group of $15-24$ years and then carriage rate decreased to less than $10 \%$ in older agegroups ${ }^{17-18}$.

Isolation of PPB was more (83.45\%) in low socioeconomic status than middle $(70.63 \%)$ and high $(25 \%)$ socioeconomic status (Table-XI). Anianson et al. (1992) reported that low socio-economic status had been identified as a risk for colonization and carriage of respiratory pathogens. Probably cramped and poor housing associated with lower access to health care are the factors ${ }^{19}$.

B. pertussis was isolated from only one child suffering from protein energy malnutrition (PEM). This child had taken only two doses of DPT vaccine. Isolation of B. pertussis indicates that the organism is in the community. Meticulous effort should be given to isolate this organism in the community level especially in malnourished children. In the present study, B. parapertussis was isolated in 5 (3.84\%) adults.
Similar high prevalence was observed by He et al. (1998). They reported that culture positivity of B. parapertussis was $3.4 \%$ in children and adults ${ }^{20}$.

In this study, B. pertussis was not isolated in culture in adults. However, $31.37 \%$ of adults were serology positive. In United State, Wright et al (1995) demonstrated that no subject was culture positive for B. pertussis, but $21 \%$ subjects met the serological criteria for pertussis infection ${ }^{21}$. The laboratory diagnosis of pertussis in adults is difficult because of the usual delay in suspicion of the disease ${ }^{22}$.

Maximum adults (43.75\%) with positive serology for IgG/IgM against B. pertussis had cough for 3-4 weeks and 14 (93.33\%) seropositive children had duration of cough for 2-3 weeks (Table-XIII). Cherry (1998) studied on prolonged cough illness in adolescents and adults and indicated that between $12 \%$ and $32 \%$ was the results of $\mathrm{B}$. pertussis infection $^{22-25}$.

Previous research in several countries had shown that pertussis is endemic among the adolescents and adults ${ }^{26-27}$. It is suggested that a universal program of adolescent and adult boosters would decrease the circulation of $\mathrm{B}$. pertussis in these age groups and possibly could lead to the elimination of the organism from the population. With the availability of vaccine, booster doses in adolescents have been introduced in Canada, Austria, Australia, France, Germany and the US, and many other countries are considering similar expansion of their immunization programs at present.

\section{Conclusion}

Potential pathogenic bacteria (PPB) present in nasopharyngeal samples were more in children than adults. The carrier rate of PPB in the nasopharynx decreased with age. However, the carrier rate of PPB was more in the low socioeconomic condition. Pertussis may be a cause of persistent cough $(>3$ weeks $)$ in adults. The affected adolescents and adults act as reservoirs of the disease to the vulnerable population of infant and children. For children presenting to primary health care center with a cough lasting for more than 2 weeks, a diagnosis of whooping cough should be considered.

\section{Reference}

1. Balows A, Duerden BI. Bacterial infections of the respiratory tract. In: Balows A, Duerden BI editors. Topley's \& Wilson's 
Microbiology and Microbial infections, $9^{\text {th }}$ edition, New York: Arnold; 1999: pp.324-325.

2. Cherry JD. From the Department of Pediatrics, David Geffen School of Medicine, University of California, Los Angeles, California, 2005: 755-756.

3. Rosenthal S, Strebel P, Cassiday P, Sanden G, Brusuelas K, Wharton M. Pertussis infection among adults during the 1993 outbreak in Chicago. J Infect Dis, 1995; $171: 1650-1652$.

4. Slack MPE. Gram Negative Coccobacilli. In: Cohen J, Powderly WG editors. Infectious Diseases, $2^{\text {nd }}$ edition. USA: Mosby Inc; 2004:pp.401-404.

5. Heininger U. Pertussis immunization in adolescents and adults. Springerlink, New York, 2008; 609: 72-97.

6. Singh M, Lingappan K. Pertussis infection in adults with persistent cough. JAMA, 1995; 273: 1044-1046.

7. Bisgard L. Children with pertussis inform the investigation of other pertussis cases among contacts. JAMA, 2004; 7: 21.

8. Lieberman D, Shleyfer E, Castel H, Terry A, Boehm IH, Delgado J. Nasopharyngeal versus oropharyngeal sampling for isolation of potential respiratory pathogens in adults. J Clin Microbiol, 2006; 44: 525-528.

9. Garcia-Rodriguez JA and Martinez MJF. Dynamics of nasopharyngeal colonization by potential respiratory pathogens. J Antimicrob Chemother, 2000; 50: 59-73.

10. Nilsson P, Ripa T. S. aureus throat colonization is more frepuent than colonization in the anterior nares. J Clin Microbiol, 2006; 44: 3334-3339.

11. Mainous AG, Hues WJ, Everett CJ, Diaz VA. Nasal carriage of $\mathrm{S}$. aureus and methicillin- resistant S. aureus in the United States, 2001-2002. Ann Fam Med, 2006; 4: 132-137.

12. Melles DC, Bogaert D. Nasopharyngeal co-colonization with S. aureus and S. pneumonia in children is bacterial genotype independent. Microbial, 2007; 153: 686-692.

13. Gunnarsson RK, Holm SE, Soderstrom M. The prevalence of potential pathogenic bacteria in nasopharyngeal samples from healthy children and adults. Scand J Prim Health Care, 1998; 16: 13-17.

14. Syrjanen RK, Kilpi TM, Kaijalainen TH, Herva EE. Nasopharyngeal carriage of $\mathrm{S}$ pneumonia in finnish children younger than 2 years old. J Infect Dis, 2001; 184: 451-459.

15. Butler JC, Shapiro ED, Carlone GM. Pneumococcal vaccines: history, current status and future directions. Am J Med, 1999;
107: 69-79.

16. Cunningham R, Matthews R, Lewendon G, Harrison S. Improved the rate of isolation of $\mathrm{N}$. meningitides by direct plating of pharyngeal swabs. J Clin Microbiol, 2001; 39: 45754576.

17 Blackwell CC, Weir DM, James VS, Todd WT, Banatvala C, Chaudhuri A et al. Secretor status, smoking and carriage of Neisseria meningitidis. Epidemiol Infect, 1990; 104: 203-209.

18. Cromer BA, Goydos J, Hackell J, Mezzatesta J, Dekker C, Mortimer EA. Unrecognized pertussis infection in adolescents. Am J Dis Child, 1993; 147: 575-577.

19. Anianson G, Alm B, Andersson B, Larsson P, Nylen O, Peterson $\mathrm{H}$ et al. Nasopharyngeal colonization during first year of life. $\mathrm{J}$ Infect Dis, 1992; 165: 38-42.

20. He Q, Edelman K, Arvilommi H, Mertsola. Protective role of immunoglobulin $\mathrm{G}$ antibodies to filamentous hemagglutinin and pertactin of B. pertussis and B. parapertussis infection. Eur J Clin Microbiol Infect Dis, 1996; 15: 793-798.

21. Wright SW, Edwards KM, Decker MD, Zeldin MH. Pertussis Infection in adults with persistent cough. JAMA, 1995; 273: 1044-1046.

22. Cherry JD. Epidemiological, clinical, and laboratory aspects of pertussis in adults. Clin Infect Dis, 1998; 28: 112-7.

23. Birkebaek NH, Kristiansen M, Seefeldt T. Bordetella pertussis and chronic cough in adults. Clin Infect Dis, 1999; 29: 1239 -1242 .

24. Gilberg S, Njamkepo E, Du Chatelet IP. Evidence of Bordetella pertussis infection in adults presenting with persistent cough in a French area with very high whole-cell vaccine coverage. J Infect Dis, 2002; 186: 415 -418.

25. Nennig ME, Shinefield HR, Edwards KM, Black SB, Fireman $\mathrm{BH}$. Prevalence and incidence of adults pertussis in urban population.JAMA, 1996; 275: 1672-1674.

26. Halperin SA, Wang EEL, Law B. Epidemiological features of pertussis in hospitalized patients in Canada, 1991-1997: report of the Immunization Monitoring Program-Active (IMPACT). Clin Infect Dis,1999; 28: 1238-1243.

27. Schmitt-Grohe S, Cherry JD, Heininger U, Uberall MA, Pineda E, Stehr K. Pertussis in German adults. Clin Infect Dis, 1995; 21: $860-866$. 